\title{
A Personal Reflection
}

\author{
Tom Nunan
}

Why on earth are you doing that - the specialty will be dead in a few years, now that we have CAT scanning.

This is what I heard in the late 1970s when I studied for the MSc in Nuclear Medicine. At that time there was only planar imaging and most of the imaging work was brain, liver and bone scanning largely for metastases and lung scans for pulmonary emboli. (For the purposes of this paper I will not cover thyroid work which has really not changed that much.) The bone scans were taken on a rectilinear scanner which technically had a better resolution than the gamma camera, but was slow and produced images that did not look anatomical so were not liked by clinicians. This is the same problem that ultrasound had, especially in the early days, when the clinicians could not understand the images (assuming they were available).

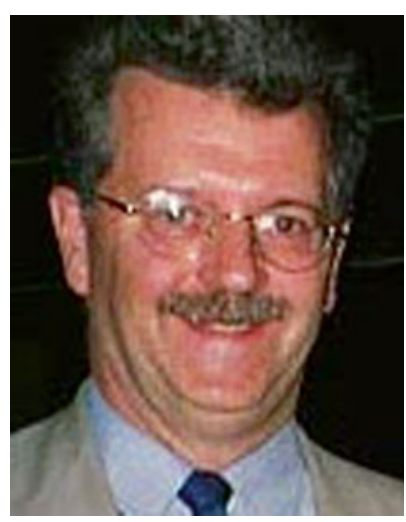

T. Nunan

Nuclear medicine, St Thomas' Hospital,

First floor, Lambeth Wing, Westminster Bridge Road, London SE1 7EH, UK

R. McCready et al. (eds.), A History of Radionuclide Studies in the UK:

50th Anniversary of the British Nuclear Medicine Society,

DOI 10.1007/978-3-319-28624-2_20 
CT brain scanning had been around since 1971. I did what were then called House Physician and Surgeon jobs at the West Middlesex Hospital in West London and I remember well the CT scanner (the first in the world) at the nearby Atkinson Morley Hospital. Quickly everyone realised the potential in diagnosis for brain diseases (not too surprising when one considers that it replaced such invasive procedures as air encephalography) and within a few years the role of CT brain scanning was established. I later looked at the early history of Xrays for an exhibition at Guy's and was struck by the similarity between the rapid rate of adoption of Xrays (Roentgenograms in those days but changed in the First World War to Xrays and Roentgengraphy changed to Radiology). But I digress.

CT brain scanning eventually replaced radionuclide blood-brain barrier scanning. In those days the resolution of everything was too poor for imaging techniques to be used for dementia. There was also the growing development of ultrasound which was to replace liver scanning, again used mainly for detecting metastases. So up went the cry that Nuclear Medicine had no future. Well, along came thallium cardiac imaging. I remember in around 1985 comparing workloads over the decade and the number of studies performed had increased, despite losing all the liver and brain scans, largely as a result of the cardiac work. In addition as an indicator of what was to come, the complexity of studies was increasing. With this came a small but useful technological advance, what were called 'large' field of view cameras. Now routine, but what it meant is it was now possible to image the whole of the chest and get in the shoulders in one image. This resulted in images which referring clinicians found easier to interpret - those of you who are still following my gist will see a trend here. Those of you who are not - well never mind!

Then along came whole body CT. (The first scanner was made by EMI and was thus called an Emiscan, then when other manufacturers entered the fray they were called CAT scans and at later date this was shortened to CT - why? I don't know). Whole body CT imaging developed in the late 80's and St Thomas' had, I believe, the second whole body EMI scanner. Again people worried that Nuclear Medicine had no future. In contrast to the CT brain scanner the use of whole body CT took some time to establish itself. I think this is because it was slow and the images were degraded by movement artefacts. Of course the first demonstration of Computerised Tomography by Oldendorf in 1961 was using isotopes and it was not developed further because the rest of technology for imaging was not ready. (Another digression, if we were to go back to 1890 and asked what do we want to develop, a functional imaging methodology for the body or 'shadow' images, which would we choose? It was only the ease with which Xray apparatus could be made and the lack of such technology that resulted in Roentgenography taking on the leading role in imaging).

Then along came SPECT. (I always wondered why do we have SPECT but not TCT or PECT?) SPECT was a bit a a game changer in cardiac studies since one now had the heart imaged in a way the cardiologists could understand and the result was a huge expansion in our cardiac workload to the point that full list cardiac studies were being performed 3-4 days per week, as opposed to one small list every 2 weeks. SPECT cardiac studies took off despite there being little evidence that they 
had better accuracy than planar images, because of the improvement in image perception. Again to digress, I remember clearly in the late 80 s, we were asked to do a thallium scan on a lady. This was a very unusual occurrence indeed. Over the years equality arrived and possibly overshot since when I retired it was my impression that female cardiac requests outnumbered male.

One of the factors that helped to develop cardiac scanning was the discovery that with conventional imaging protocols, myocardium that did not take up Thallium could be shown to be viable post revascularisation rather than as conventional wisdom had it, infarcted. Indeed I remember cardiac surgeons saying as much but a paper in the NEJM showed these anecdotes to be correct. The above statement might sound counterintuitive but the threat this raised resulted in two things happening. The first was to relook at the thallium protocol. It was then realised that the one day protocol with a stress image first and a redistribution image several hours later was a compromise between convenience, dose and accuracy. The correct way to do the study was to have a proper rest image. This leads to the second point which was that thallium for a 2 day injection protocol had a rather high radiation dose. Thus the manufacturers developed ${ }^{99 \mathrm{~m}} \mathrm{Tc}$ tracers to do the job. This had an added advantage since modern cameras are made with ${ }^{99 \mathrm{~m}} \mathrm{Tc}$ in mind and are more suited to ${ }^{99 \mathrm{~m}} \mathrm{Tc}$ than $\mathrm{Tl}^{201}$. To yet another digression, it is always useful to look at things from the basics, often there are compromises made which will always result in sacrificing something. I am reminded of the endless papers on the measurement of GFR where there was (and maybe still is) compromise in the number of samples taken versus the convenience to the patient.

Things got quiet then in terms of development, MRI was coming on the scene, CTPA was supposed to replace lung scanning. Yet again the cry went up - 'Nuclear Medicine has no future'. Perhaps it is this threat that gets people looking hard at what they do and coming up with advances.

But to counter MRI we had PET scanning then PET/CT. The real development that allowed these to happen was the development of computer power. Many of those of us who are 'more senior' will remember stories of studies being performed, the data loaded into the computer and then everyone went home for the night and if they were lucky it would be ready to look at in the morning. I also remember the South-East region getting some gamma cameras which could either acquire an image or process it, not both at the same time. Think how it is now - even the most complex PET/CT study is ready before the patient leaves the room.

I think we are eventually arriving at a point where the advantages of functional imaging are becoming main stream, all as a result of hybrid images and the excellent image quality that they provide. Our referring clinicians can easily interpret the images to see and more to the point, understand what we are talking about in the report. (Beware of my predictions, I once was asked by GE what I thought of double headed cameras and said I thought they were too expensive for people to buy. But as was once said "All technology is too expensive." When I looked on Google to see who said this, I could not find it, but I note that UEFA President Platini is saying it in relation to goal line cameras, so it is bound to come). 
So over my career, Nuclear Medicine changed out of all recognition. Few of the types of study that I started with are routine now, they have either been replaced by other imaging modalities (liver scans), been significantly modified (SPECT bone and lung scans) or are completely new (sentinel node and PET/CT). Thus although people may say again 'don't consider Nuclear Medicine as a career because it will not last' - they are and have been wrong!

Open Access This chapter is distributed under the terms of the Creative Commons AttributionNoncommercial 2.5 License (http://creativecommons.org/licenses/by-nc/2.5/) which permits any noncommercial use, distribution, and reproduction in any medium, provided the original author(s) and source are credited.

The images or other third party material in this chapter are included in the work's Creative Commons license, unless indicated otherwise in the credit line; if such material is not included in the work's Creative Commons license and the respective action is not permitted by statutory regulation, users will need to obtain permission from the license holder to duplicate, adapt or reproduce the material. 